\title{
Dynamical modelling of a genetic algorithm
}

\author{
E.J. Solteiro Pires ${ }^{\mathrm{a}, *}$, J.A. Tenreiro Machado ${ }^{\mathrm{b}}$, P.B. de Moura Oliveira ${ }^{\mathrm{a}, \mathrm{c}}$ \\ ${ }^{a}$ Univ. Trás-os-Montes e Alto Douro, Dep. de Engenharia Electrotécnica, 5000-911 Vila Real, Portugal \\ ${ }^{\mathrm{b}}$ Instituto Superior de Engenharia do Porto, Dep. de Engenharia Electrotécnica, Rua Dr. António Bernadino de Almeida, \\ 4200-072 Porto, Portugal \\ ${ }^{\mathrm{c} C e n t r o ~ d e ~ E s t u d o s ~ T e c n o l o ́ g i c o s, ~ d o ~ A m b i e n t e ~ e ~ d a ~ V i d a, ~ 5000-911 ~ V i l a ~ R e a l, ~ P o r t u g a l ~}$
}

Received 12 April 2005; received in revised form 29 October 2005; accepted 6 December 2005

Available online 9 March 2006

\begin{abstract}
This work addresses the signal propagation and the fractional-order dynamics during the evolution of a genetic algorithm (GA). In order to investigate the phenomena involved in the GA population evolution, the mutation is exposed to excitation perturbations during some generations and the corresponding fitness variations are evaluated. Three distinct fitness functions are used to study their influence in the GA dynamics. The input and output signals are studied revealing a fractional-order dynamic evolution, characteristic of a long-term system memory.
\end{abstract}

(C) 2006 Elsevier B.V. All rights reserved.

Keywords: Genetic algorithm; Signal processing; Dynamic; Fractional-order

\section{Introduction}

In the last twenty years genetic algorithms (GAs) have been applied in a plethora of fields such as in image processing, pattern recognition, speech recognition, control, system identification, optimization, planning and scheduling [1].

Fractional calculus (FC) is a natural extension of the classical mathematics. In fact, since the beginning of theory of differential and integral calculus, several mathematicians investigated the calculation of non-integer order derivatives and integrals. Nevertheless, the application of FC has been scarce

\footnotetext{
*Corresponding author.

E-mail addresses: epires@utad.pt (E.J. Solteiro Pires), jtm@isep.ipp.pt (J.A. Tenreiro Machado), oliveira@utad.pt (P.B. de Moura Oliveira).
}

until recently, but the advances in the theory of chaos motivated a renewed interest in this field.

The fundamental aspects of the FC theory are addressed in [2-5]. In what concerns the FC application we can mention research on viscoelasticity/damping, chaos/fractals, biology, electronics, signal processing, diffusion and wave propagation, percolation, modelling, control and irreversibility [6-19,23].

Bearing these ideas in mind, this paper analyzes the system signal evolution and the fractional-order dynamics in the population of a GA-based optimization. The article is organized as follows. Section 2 introduces the problem and the GA method for its resolution. Based on this formulation, Section 3 presents the results for several simulations involving different working conditions and studies the resultant dynamic phenomena. Section 4 present the same simulation with others fitness functions and 
compare the results. Finally, Section 5 outlines the main conclusions.

\section{The optimization GA}

This section presents the optimization GA used in the study of the dynamic system. To investigate the influence of the fitness function in the GA, are used three simple functions $\left\{f_{A}, f_{B}, f_{C}\right\}$, each at a time,

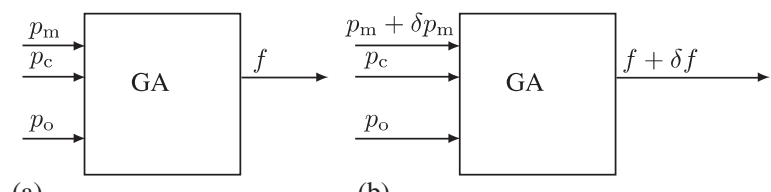

Fig. 1. System dynamics. (a) Initial system, (b) perturbed system.
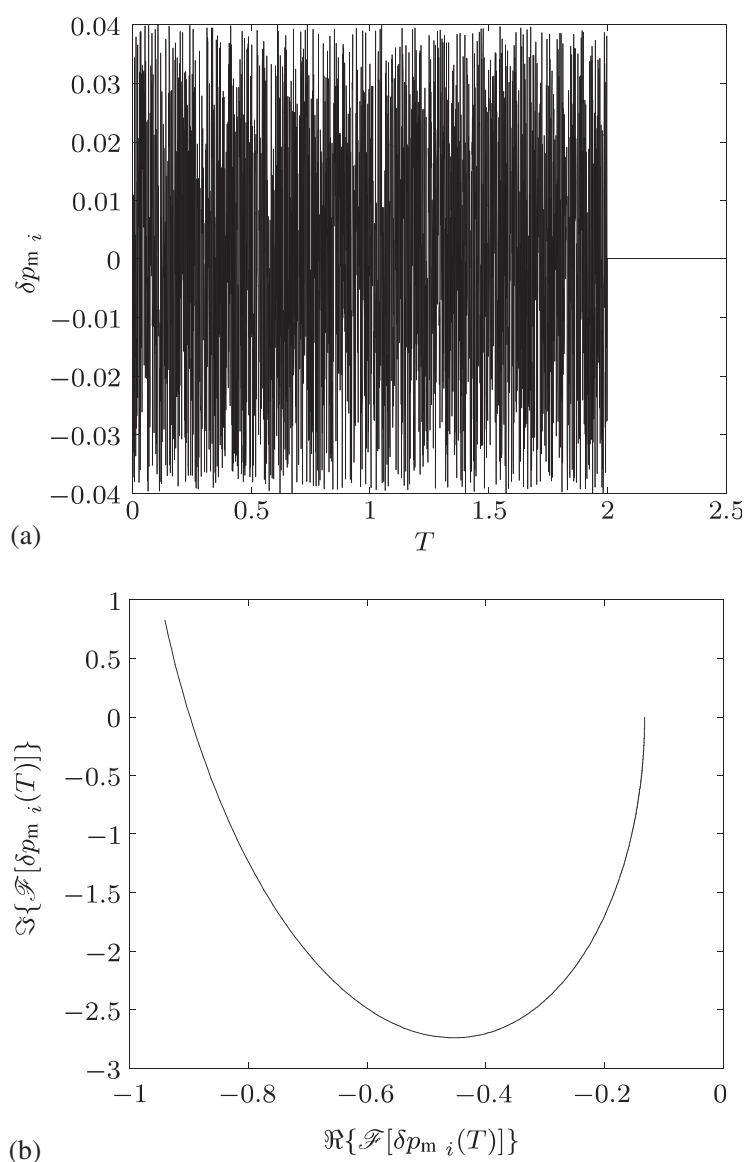

Fig. 2. Input signal $\delta p_{\mathrm{m}}$ perturbation over $T_{\mathrm{exc}}=2$ generations with seed $i=1\left(\Delta p=0.04\right.$, fitness $\left.f_{A}\right)$. (a) Generation domain, (b) polar diagram. given by the expressions

$$
\begin{aligned}
& f_{A}(b)=1+|b-41|, \\
& f_{B}(b)=1+|b-41|^{2}, \\
& f_{C}(b)=2-\operatorname{sinc}(b-41), \\
& b=\left\{b_{1}, b_{2}, b_{3}, \ldots, b_{l}\right\} .
\end{aligned}
$$

The objective consists on minimizing each function that depends on the parameter $b \in[-50000,50000]$. In the experiments, involving the three test functions, it is adopted a binary Gray code, with the string length of $l=24$ bits to represent the solutions of the population (2) while having an identical initial population. The same applies for the genetic parameters, namely (i) a 50-population GA, (ii) an execution during 200 generations, (iii) a rank selection having simple crossover and mutation with probabilities $p_{\mathrm{c}}=0.8$ and $p_{\mathrm{m}}=0.05$, respectively. Moreover, the best solution of each generation is always passed to the next generation. The
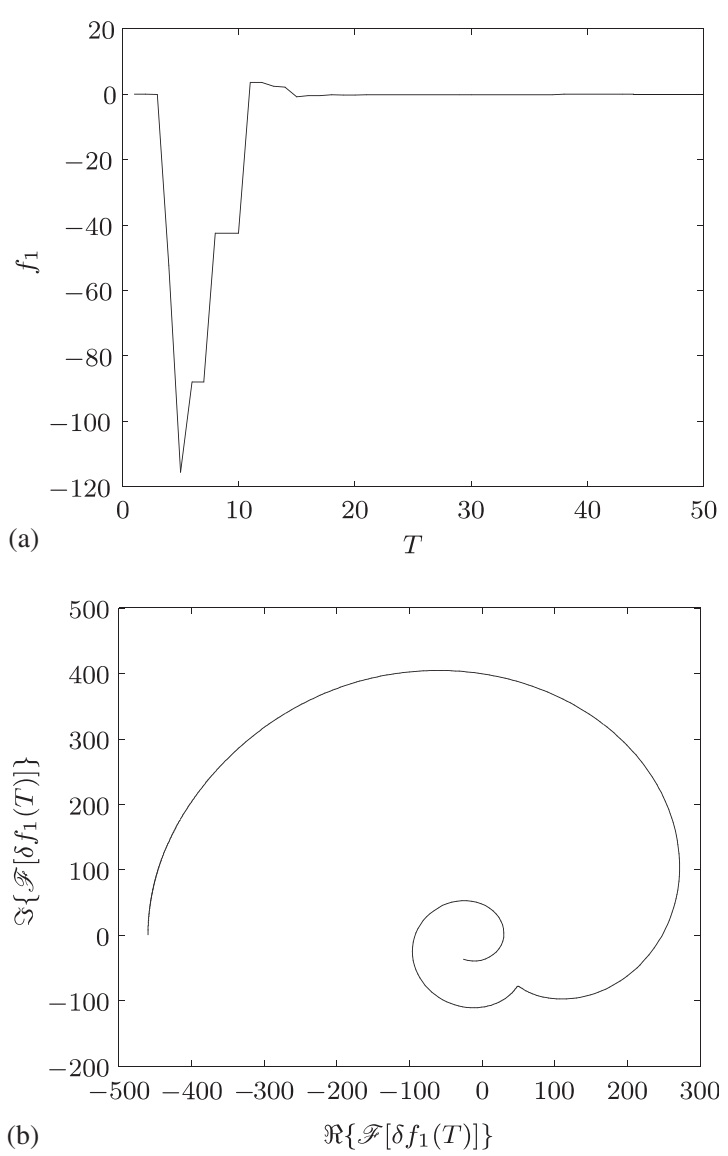

Fig. 3. Output variation $\delta f(T)$ for the input excitation over $T_{\text {exc }}=2$ generations with seed $i=1\left(\Delta p=0.04\right.$, fitness $\left.f_{A}\right)$. (a) Generation domain, (b) polar diagram. 
influence of several factors can be analyzed in order to study the dynamics of the GA, particularly the probabilities. This influence can be distinct according to the type of selection, elitism, fitness function and string length used in the GA. In this work,

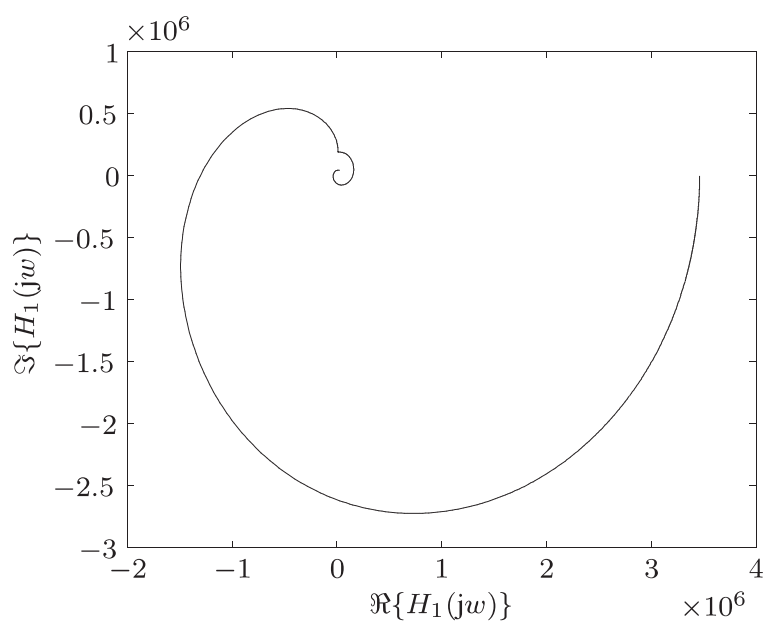

Fig. 4. Transfer function $H_{1}(\mathrm{j} w)$ using seed $i=1 \quad\left(T_{\mathrm{exc}}=2\right.$, $\Delta p=0.04$, fitness $f_{A}$ ).

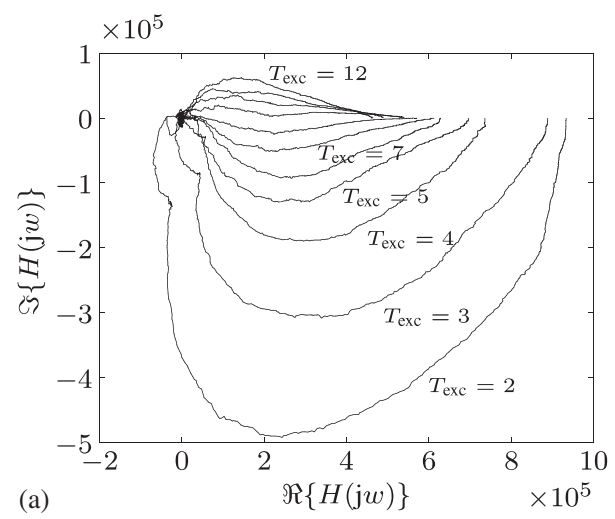

across each test function experiment, it is changed only the initial seed of the mutation probability noise that is added to $p_{\mathrm{m}}$.

\section{Evolution, signal propagation and fractional-order dynamics}

This section studies the dynamical phenomena involved in the signal propagation within the GA population. In this perspective, small amplitude perturbations are superimposed over biasing signals of the GA system and its influence upon the population fitness is evaluated. The experiments reveal a fractional-order dynamics capable of being described by systems theory tools.

\subsection{Introduction to fractional calculus}

Since the foundation of the differential calculus the generalization of the concept of derivative and integral to a non-integer order $\alpha$ has been the subject of distinct approaches. Due to this reason there are several alternative definitions of fractional

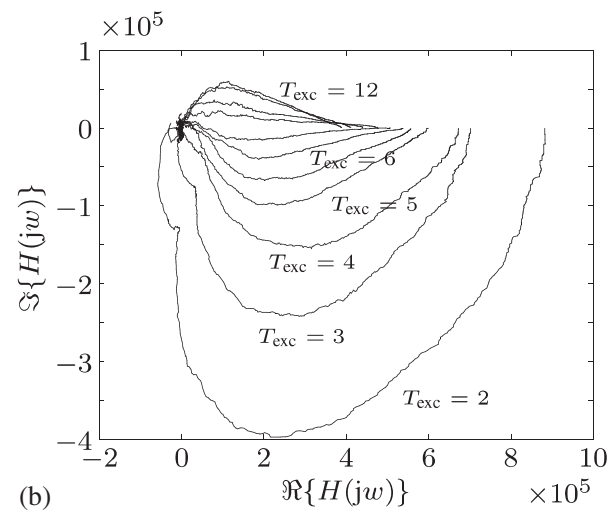

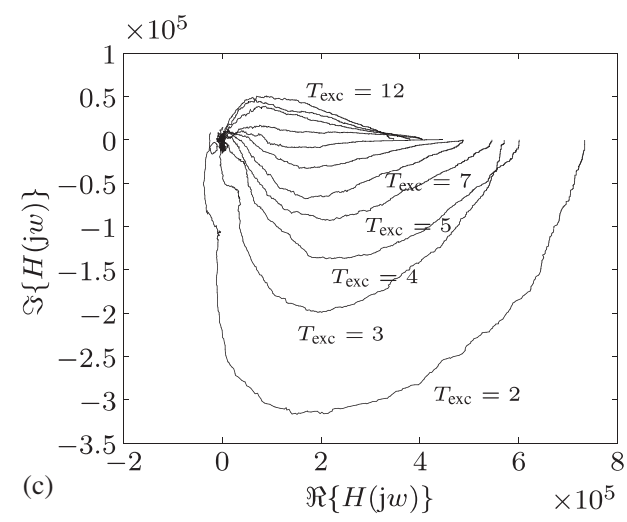

Fig. 5. Polar diagrams of $H_{A}(\mathrm{j} w)$ for $\Delta p=\{0.03,0.04,0.05\}, n=1701$. (a) Polar diagram with $\Delta p=0.03$, (b) polar diagram with $\Delta p=0.04$, (c) polar diagram with $\Delta p=0.05$. 
derivatives. For example, the Laplace definition of a derivative of fractional order $\alpha \in \mathbb{C}$ of the signal $x(t), D^{\alpha}[x(t)]$, is a 'direct' generalization of the classic integer-order scheme yielding to Eq. (3). This means that frequency-based analysis methods have a straightforward adaptation (for zero initial conditions):

$\mathscr{L}\left\{D^{\alpha}[x(t)]\right\}=s^{\alpha} X(s)$.
An alternative approach, based on the concept of fractional differential, is the Grünwald-Letnikov definition given by Eq. (4)

$$
\begin{aligned}
D^{\alpha}[x(t)]= & \lim _{h \rightarrow 0}\left[\frac{1}{h^{\alpha}} \sum_{k=0}^{+\infty}(-1)^{k}\right. \\
& \left.\times \frac{\Gamma(\alpha+1)}{\Gamma(k+1)(\alpha-k+1)} x(t-k h)\right] .
\end{aligned}
$$
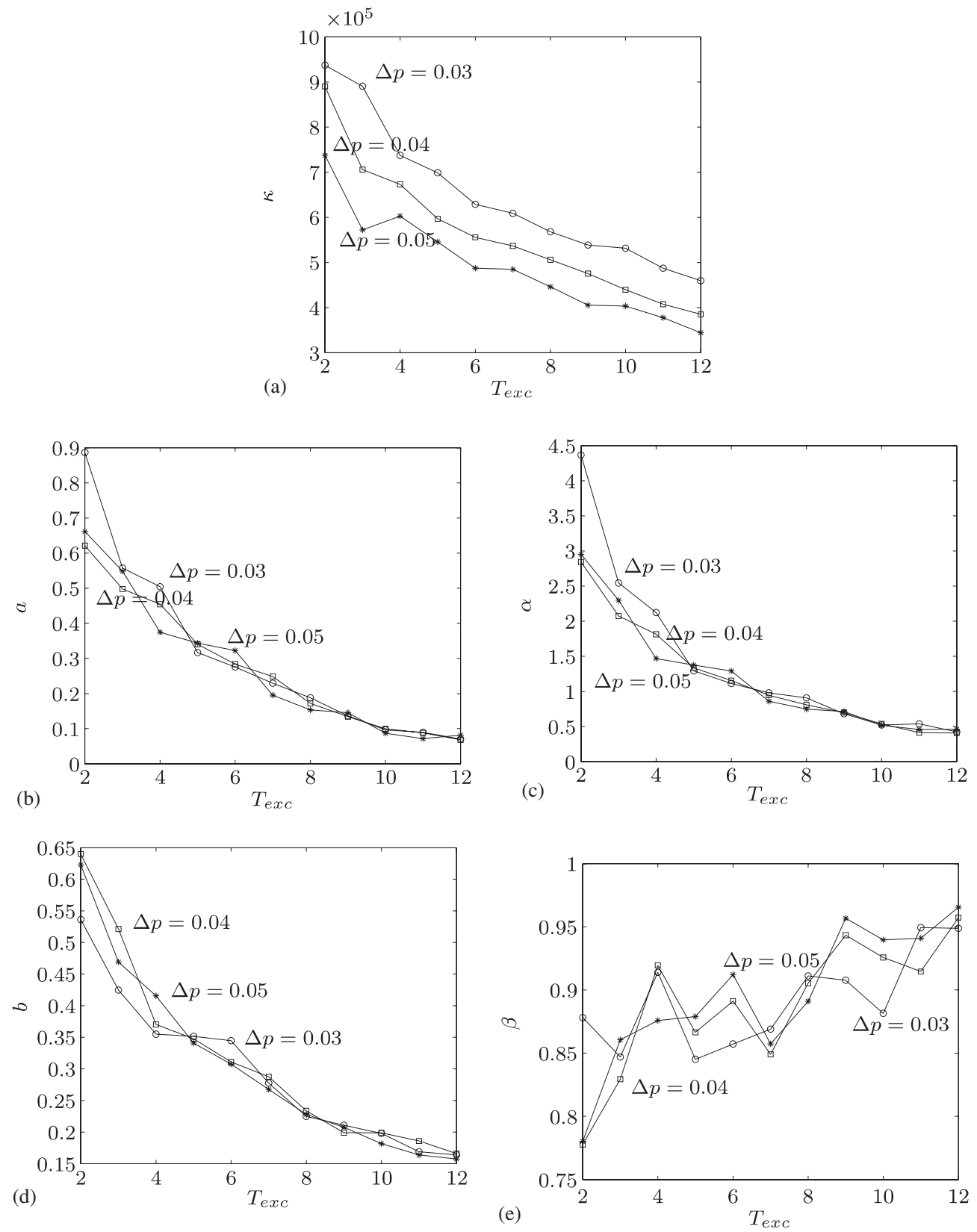

Fig. 6. Estimated gain, poles and fractional-orders vs. $T_{\text {exc }}$ for fitness $f_{A}$. (a) Gain $\kappa$ vs. $T_{\text {exc }}$, (b) pole $a$ vs. $T_{\text {exc }}$, (c) pole fractional-order $\alpha$ vs. $T_{\text {exc }}$, (d) pole $b$ vs. $T_{\text {exc }}$, (e) pole fractional-order $\beta$ vs. $T_{\text {exc }}$. 
An important property revealed by this equation is that while an integer-order derivative implies just a finite series, the fractional-order derivative requires an infinite number of terms. This means that integer derivatives are 'local' operators in opposition with fractional derivatives that have, implicitly, a 'memory' of all past events.

The characteristics revealed by fractional-order models make this mathematical tool well suited to describe phenomena such as irreversibility and chaos because of its inherent memory property. In this line of thought, the propagation of perturbations and the appearance of long-term dynamic phenomena in a population of individuals subjected to an evolutionary process seem to be a case where FC tools fit adequately.

\subsection{The $G A$ dynamics}

In this section the GA system is stimulated by perturbing the mutation probability, $p_{\mathrm{m}}$, through a white noise signal, $\delta p_{\mathrm{m}}$, and the corresponding

Table 1

Parameters of $\gamma_{i}, i=\{1,2\}$ for $\{\kappa, a, \alpha, b, \beta\}$ approximation of $f_{A}$ optimization function

\begin{tabular}{|c|c|c|c|c|c|c|c|c|c|c|}
\hline \multirow[t]{2}{*}{$\Delta p$} & \multicolumn{2}{|l|}{$\kappa$} & \multicolumn{2}{|l|}{$a$} & \multicolumn{2}{|l|}{$\alpha$} & \multicolumn{2}{|l|}{$b$} & \multicolumn{2}{|l|}{$\beta$} \\
\hline & $\gamma_{1}\left(10^{5}\right)$ & $\gamma_{2}$ & $\gamma_{1}$ & $\gamma_{2}$ & $\gamma_{1}$ & $\gamma_{2}$ & $\gamma_{1}$ & $\gamma_{2}$ & $\gamma_{1}$ & $\gamma_{2}$ \\
\hline 0.03 & 10.0 & -0.40 & 2.90 & -1.40 & 10.86 & 0.99 & 0.93 & -0.67 & 0.83 & 0.04 \\
\hline 0.04 & 10.0 & -0.44 & 2.12 & -1.26 & 7.42 & -1.11 & 1.14 & -0.76 & 0.75 & 0.09 \\
\hline 0.05 & 9.7 & -0.39 & 2.50 & -1.31 & 7.23 & -1.10 & 1.16 & -0.79 & 0.75 & 0.10 \\
\hline
\end{tabular}
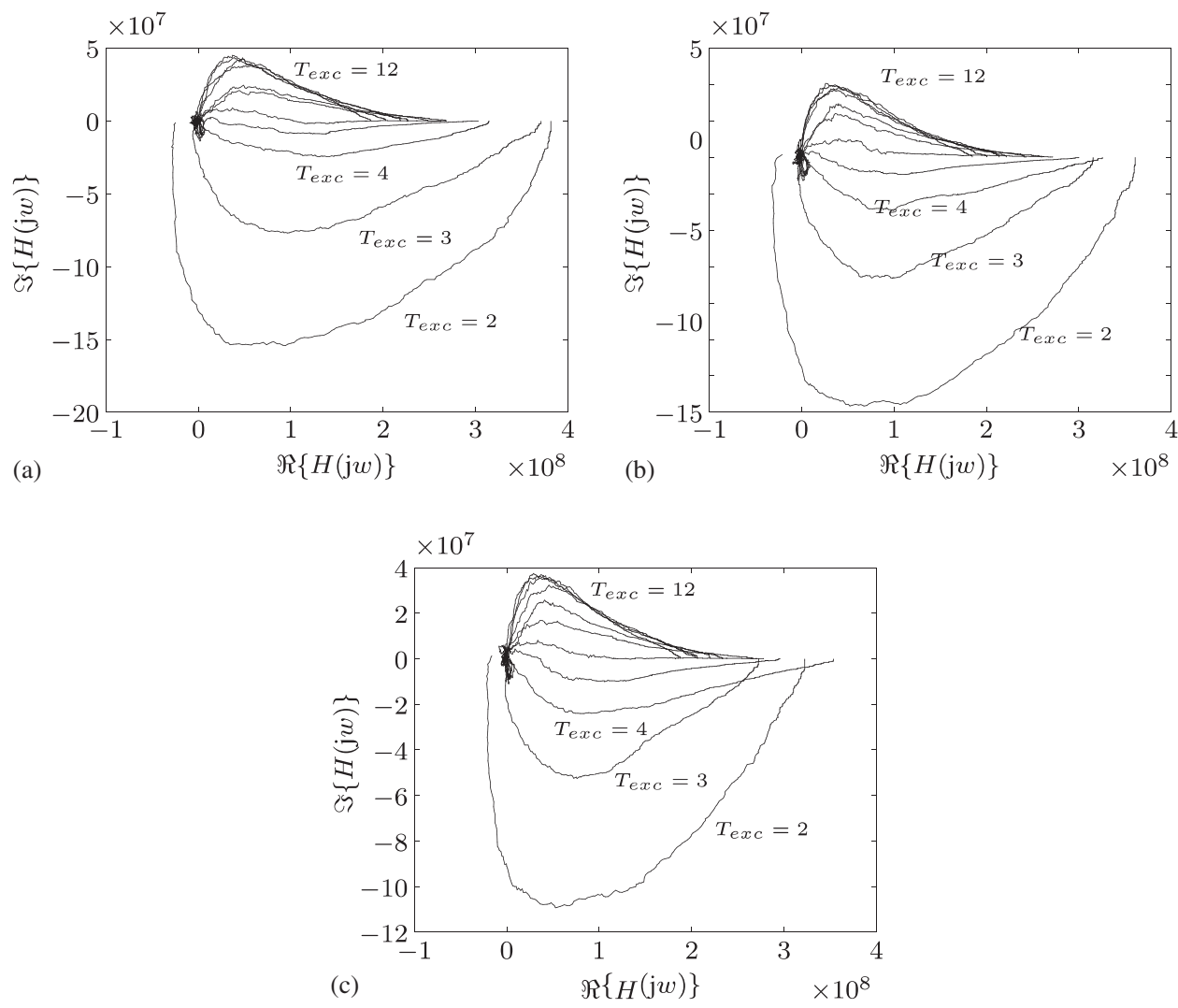

Fig. 7. Polar diagrams of $H_{B}(\mathrm{j} w)$ for $\Delta p=\{0.03,0.04,0.05\}, n=1701$. (a) Polar diagram with $\Delta p=0.03$, (b) polar diagram with $\Delta p=0.04$, (c) polar diagram with $\Delta p=0.05$. 

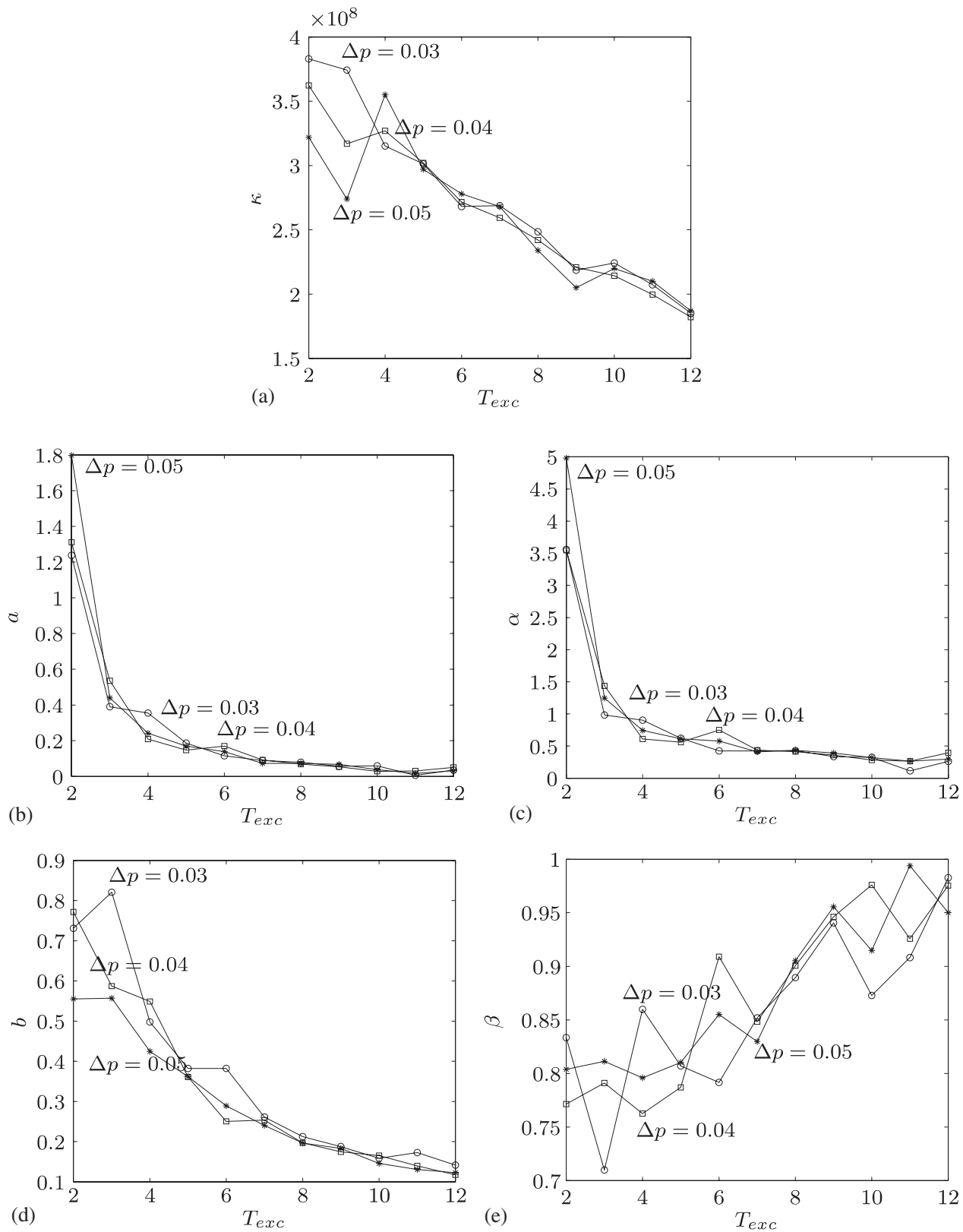

Fig. 8. Estimated gain, poles and fractional-orders vs. $T_{\text {exc }}$ for fitness $f_{B}$. (a) Gain $\kappa$ vs. $T_{\text {exc }}$, (b) pole $a$ vs. $T_{\text {exc }}$, (c) pole fractional-order $\alpha$ vs. $T_{\text {exc }}$, (d) pole $b$ vs. $T_{\text {exc }}$, (e) pole fractional-order $\beta$ vs. $T_{\text {exc }}$.

Table 2

Parameters of $\gamma_{i}, i=\{1,2\}$ for $\{\kappa, a, \alpha, b, \beta\}$ approximation of $f_{B}$ optimization function

\begin{tabular}{|c|c|c|c|c|c|c|c|c|c|c|}
\hline \multirow[t]{2}{*}{$\Delta p$} & \multicolumn{2}{|l|}{$\kappa$} & \multicolumn{2}{|l|}{$a$} & \multicolumn{2}{|l|}{$\alpha$} & \multicolumn{2}{|l|}{$b$} & \multicolumn{2}{|l|}{$\beta$} \\
\hline & $\gamma_{1}\left(10^{8}\right)$ & $\gamma_{2}$ & $\gamma_{1}$ & $\gamma_{2}$ & $\gamma_{1}$ & $\gamma_{2}$ & $\gamma_{1}$ & $\gamma_{2}$ & $\gamma_{1}$ & $\gamma_{2}$ \\
\hline 0.03 & 6.0 & -0.40 & 6.39 & -2.26 & 6.87 & -1.44 & 2.02 & -1.05 & 0.70 & 0.11 \\
\hline 0.04 & 5.0 & -0.38 & 4.66 & -2.03 & 5.65 & -1.25 & 1.93 & -1.09 & 0.66 & 0.15 \\
\hline 0.05 & 4.0 & -0.30 & 6.83 & -2.27 & 7.39 & -1.40 & 1.42 & -0.95 & 0.70 & 0.12 \\
\hline
\end{tabular}


population fitness modification $\delta f$ is evaluated, see Fig. 1. The crossover and other probability signals used, $p_{\mathrm{c}}$ and $p_{\mathrm{o}}$, remain unchanged. Therefore, the variation of the mutation probability and the resulting fitness modification on the GA population, during the evolution, can be viewed as the system inputs and output signals varying during the successive generations. This analysis is evaluated using several experiments with different seeds for a small amplitude white noise perturbation signal. All the other seeds remain unchanged.

In this perspective, a white noise signal $\delta p_{\mathrm{m}}(T)$ is added to the mutation probability $p_{\mathrm{m}}$ of the strings during a time period $T_{\text {exc }}$ and the new mutation probability $p_{\mathrm{n}}$ is calculated by Eq. (5) where $\eta$ is the white noise signal with maximum amplitude $\pm \Delta p$

$p_{\mathrm{n}}= \begin{cases}0 & \text { if } p_{\mathrm{m}}+\eta(\Delta p)<0, \\ 1 & \text { if } p_{\mathrm{m}}+\eta(\Delta p)>1, \\ p_{\mathrm{m}}+\eta(\Delta p) & \text { otherwise. }\end{cases}$

Consequently, the input signal, at generation $T$, is the difference between the two cases, that is $\delta p_{\mathrm{m}}(T)=p_{\mathrm{n}}(T)-p_{\mathrm{m}}(T)$. On the other hand, the output signal is the difference in the population fitness with and without noise, that is $\delta f(T)=$ $f_{\mathrm{n}}(T)-f(T)$.

Fig. 2 shows the input signal $\delta p_{\mathrm{m}}(T)$, with seed $i=1$, in the generation domain and the corresponding polar diagram, for $f_{A}, \Delta p=0.04$ and $T_{\mathrm{exc}}=2$, where $\mathscr{F}\left[\delta p_{\mathrm{m}}(T)\right]$ represents the Fourier transform of the signal perturbation. Fig. 3 shows the corresponding output signal variation $\delta f(T)$.

Once having de Fourier description of the input and output signals it is possible to calculate the corresponding transfer function (6) for seed $i$

$H_{i}(\mathrm{j} w)=\frac{\mathscr{F}\left\{\delta f_{i}(T)\right\}}{\mathscr{F}\left\{\delta p_{\mathrm{m} i}(T)\right\}}, \quad i=\{1, \ldots, n\}$.

The transfer function $H_{i}(\mathrm{j} w)$, with seed $i=1$, between the input and output signals, is depicted in Fig. 4.

After repeating for all seeds a 'representative' transfer function is obtained by using the median of the statistical sample [20,21] of $n$ experiments.

\subsection{Transfer function identification}

In this section the median of the numerical system transfer function, Fig. 5, is approximated by analytical expressions with gain $k \in \mathbb{R}^{+}$and two poles $(a, b) \in \mathbb{R}^{+}$of fractional orders $(\alpha, \beta) \in \mathbb{R}^{+}$, respectively, given by Eq. (7) where $l=A$ for function $f_{A}$

$G_{l}(\mathrm{j} w)=\frac{\kappa}{[(\mathrm{j} w / a)+1]^{\alpha}\left[(-\mathrm{j} w / b)^{\beta}+1\right]}$.

Note that expression (7) is a transfer function adopting left and right half-plane poles $a$ and $b$, respectively.

In order to estimate the transfer function parameters, an identification GA adopting a real string is executed with the representation $\{k, a, b, \alpha, \beta\}$. The identification GA is executed during $T_{\text {ide }}=600$ generations with a 100 strings population. It is used

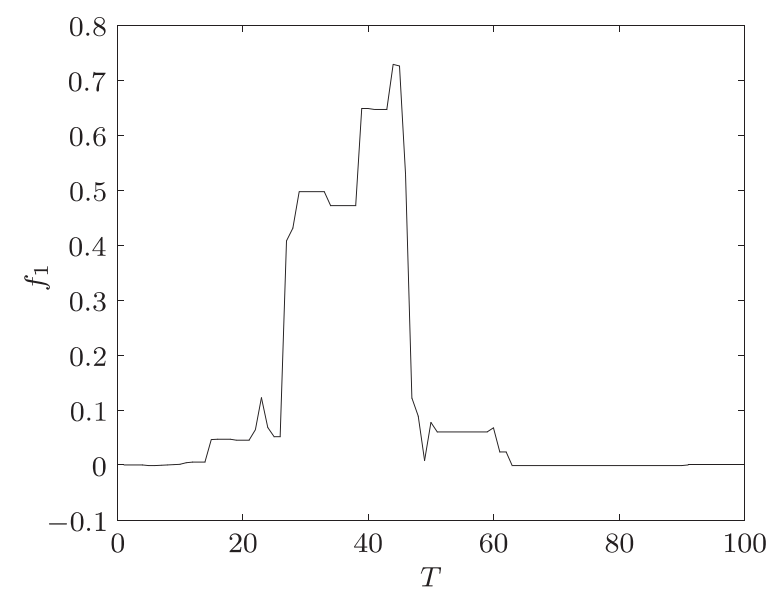

Fig. 9. Output variation $\delta f(T)$ for fitness $f_{C}$ and for the input excitation over $T_{\mathrm{exc}}=2$ generations with seed $i=1(\Delta p=0.04)$.

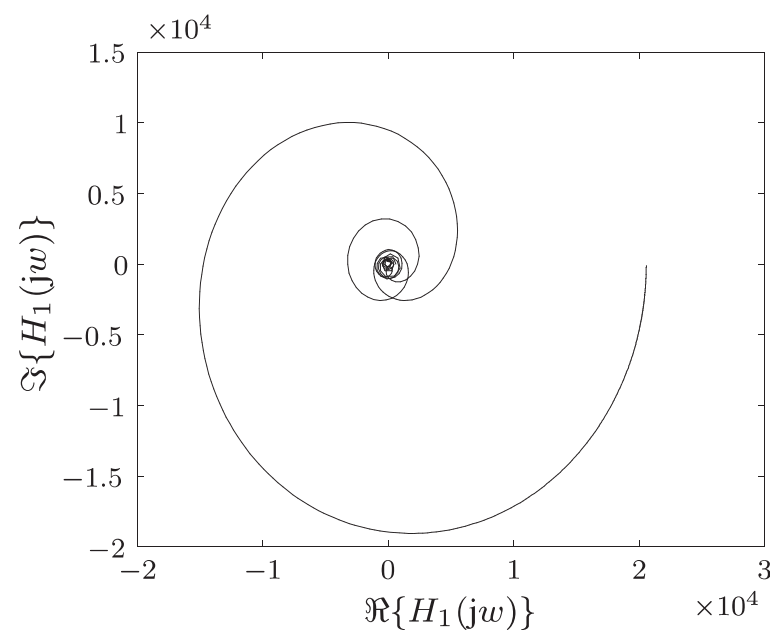

Fig. 10. Transfer function $H_{1}(\mathrm{j} w)$ for fitness $f_{C}$ and using seed $i=1\left(T_{\text {exc }}=2, \Delta p=0.04\right)$. 
the simulated binary crossover [22] and, when one mutation occurs, the corresponding value $\left\{x_{1}, \ldots, x_{5}\right\} \equiv\{k, a, b, \alpha, \beta\}$ is changed according with the equations:

$x_{i+1}=10^{u_{i}} x_{i}$,

$u_{i} \sim \mathrm{U}\left[-\varepsilon_{i},+\varepsilon_{i}\right]$,

where $u_{i}$ is a random number generated through the uniform probability distribution $\mathrm{U}$ and $\varepsilon_{i}$ is fixed according with the range of estimation.

The fitness function $f_{\text {ide }}$ measures the distance between the median $H\left(\mathrm{j} w_{k}\right)$ and $G\left(\mathrm{j} w_{k}\right)$ :

$f_{\text {ide }}=\sum_{k=1}^{n f}\left\|H\left(\mathrm{j} w_{k}\right)-G\left(\mathrm{j} w_{k}\right)\right\|$,

where $H$ represents the median of the $n$ transfer functions resulting for each different seed, $n f$ is the total number of sampling points and $w_{k}$, $k=\{1, \ldots, n f\}$ is the corresponding vector of frequencies.

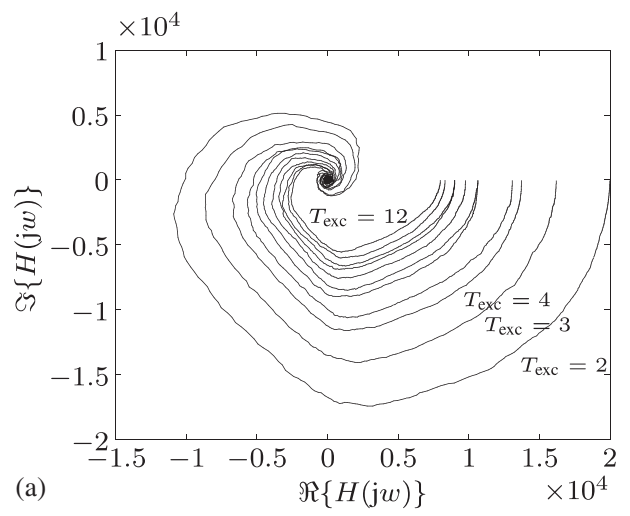

Since the optimization GA has a stochastic dynamics, each execution of the GA, with a different noise seed, leads to a different transfer function. Consequently, in order to obtain a numerical convergence [20] are performed $n=$ 1701 perturbation experiments, for each optimization function, with different seeds for the white noise signal, $\delta p_{\mathrm{m}}(T)$ (all the other seeds for $p_{\mathrm{m}}, p_{\mathrm{c}}$ and selection remains unchanged). So, the transfer function of the optimization GA dynamics is evaluated by computing the Fourier transform (FT) for each pair of input and output signals. After that, the medians of the transfer functions calculated previously (i.e., for each real and imaginary part and for each frequency) are taken as the final part of the numerical transfer function $H(\mathrm{j} w)$ (see Fig. 5).

For evaluating the influence of the excitation period $T_{\text {exc }}$ several simulations are performed ranging from $T_{\text {exc }}=2$ to $T_{\text {exc }}=12$ generations. The relation between the transfer function parameters and $T_{\text {exc }}$ are shown in Fig. 6 .
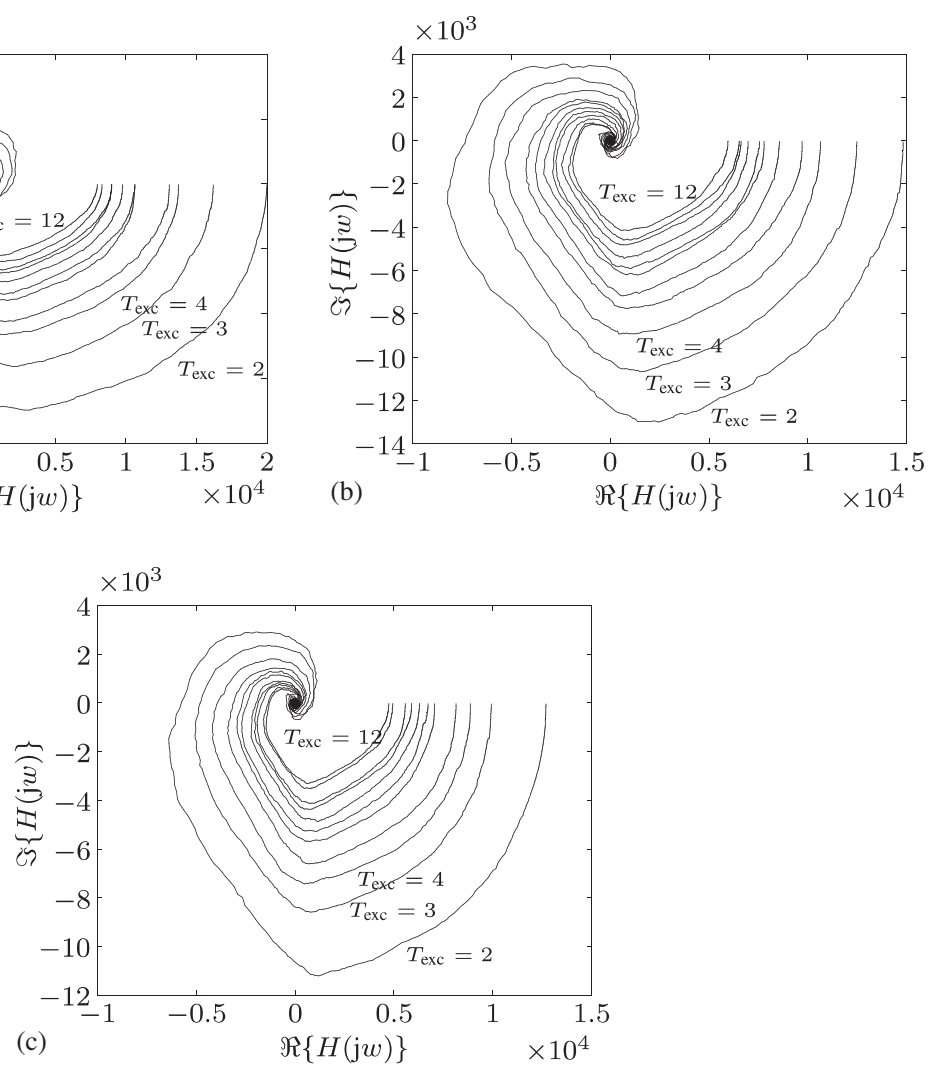

Fig. 11. Polar diagrams of $H(\mathrm{j} w)$ for fitness $f_{C}(b)$ and $\Delta p=\{0.03,0.04,0.05\}, n=1701$. (a) Polar diagram with $\Delta p=0.03$, (b) polar diagram with $\Delta p=0.04$, (c) polar diagram with $\Delta p=0.05$. 
The charts of $\{\kappa, a, b, \alpha, \beta\}$ can be approximated through Eq. (10) leading to the parameters of Table 1.

$\{\kappa, a, \alpha, b, \beta\} \simeq \gamma_{1}\left(T_{\text {exc }}\right)^{\gamma_{2}}$.

These results reveal that the transfer function parameters $\{\kappa, a, \alpha, b, \beta\}$ vary with a power law vs. the excitation time $T_{\text {exc }}$. Moreover, all the parameters have a strong dependence with $T_{\text {exc }}$ on the transfer function. Furthermore, $\kappa$ varies with $\Delta p$ and $p_{\mathrm{m}}$ while $\{a, \alpha, b, \beta\}$ have a low dependence with $\Delta p$ and $p_{\mathrm{m}}$.
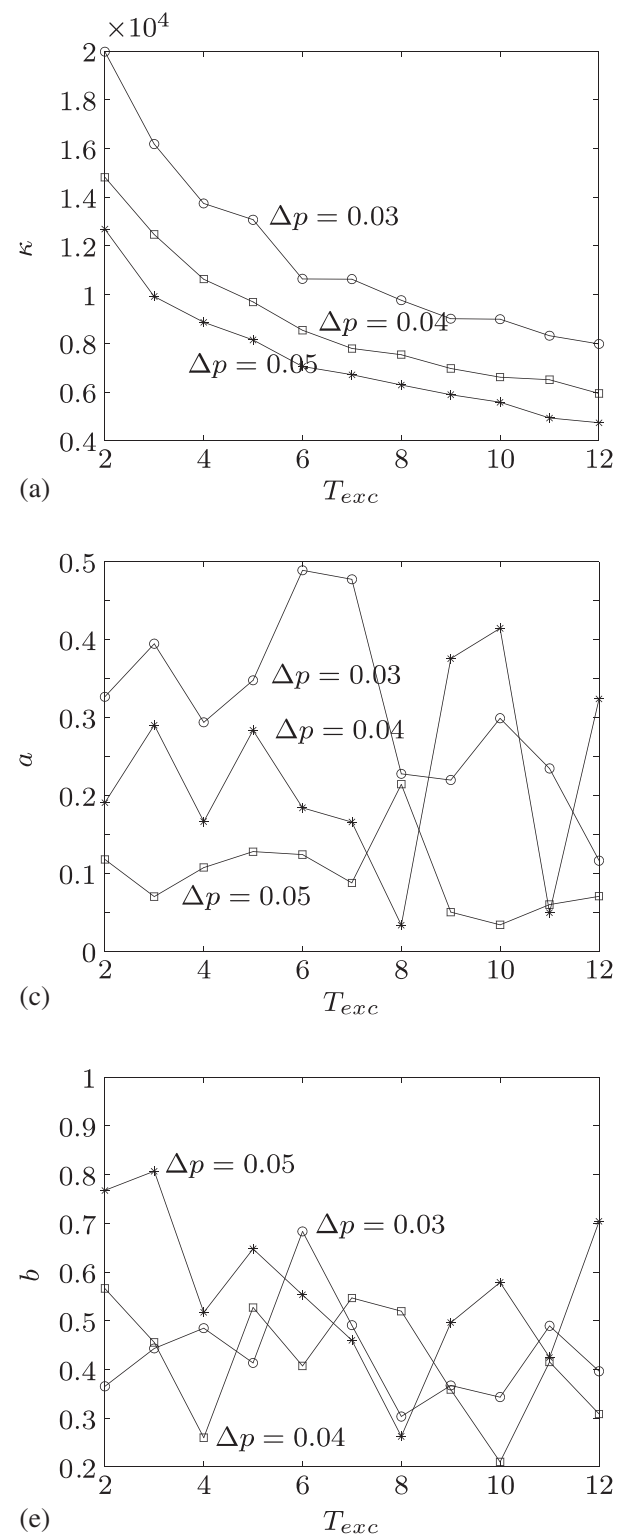

By enabling the zero/pole order to vary freely, we get non-integer values for $\alpha$ and $\beta$. Alternatively, the adoption of an integer-order transfer function would lead to a larger number of zero/poles to get the same quality in the analytical fitting to the numerical values. The 'requirement' of fractionalorder models, in opposition with the classical case of integer models, is a well-known discussion and even nowadays final conclusions are not clear since it is always possible to approximate a fractional frequency response through an integer one as long as we make use of a larger number of zeros and
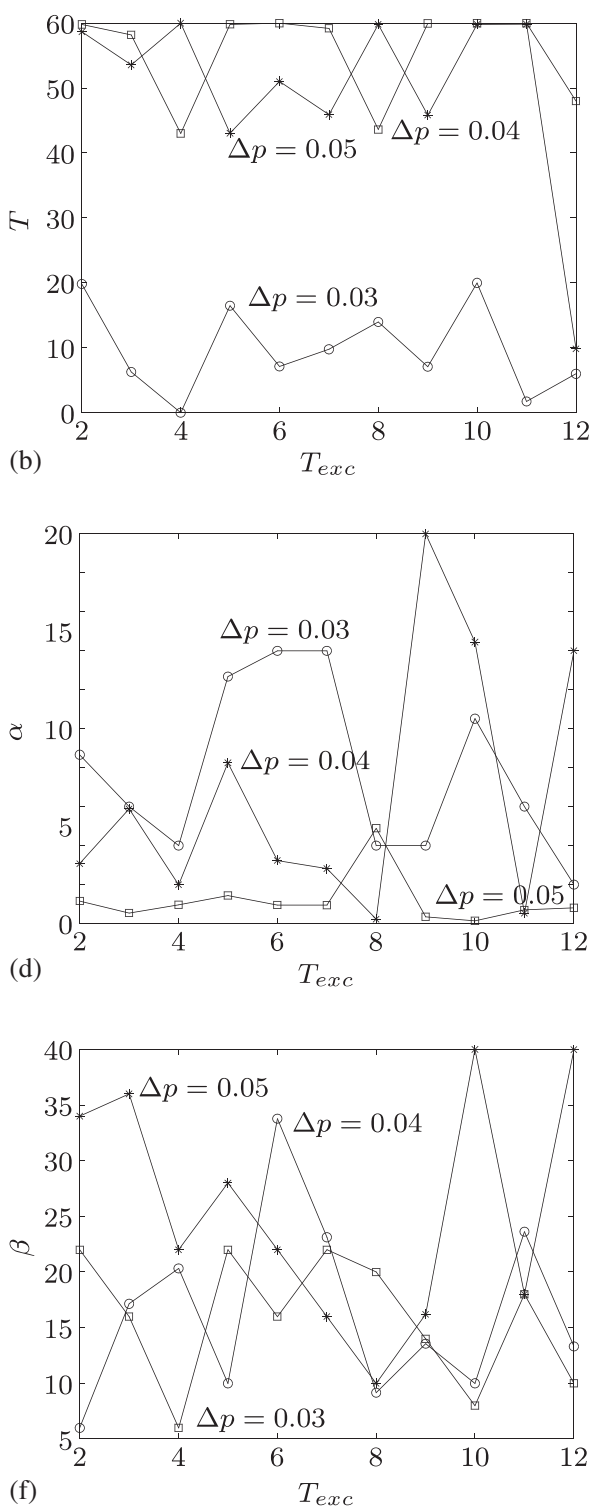

Fig. 12. Estimated parameters vs. $T_{\text {exc }}$ for fitness $f_{C}$. (a) Gain $\kappa$ vs. $T_{\text {exc }}$, (b) delay $T$ vs. $T_{\text {exc }}$, (c) pole $a$ vs. $T_{\text {exc }}$, (d) pole fractional-order $\alpha$ vs. $T_{\text {exc }}$, (e) pole $b$ vs. $T_{\text {exc }}$, (f) pole fractional-order $\beta$ vs. $T_{\text {exc }}$. 
poles. Nevertheless, the present experiments constitute a complementary point of view towards the adoption of FC.

\section{Experiments with the fitness functions $f_{B}$ and $f_{C}$}

In this section is presented the polar diagram and the correspondent parameters approximation when is used the $f_{B}$ and $f_{C}$ optimization functions. The experiments are carried out in the same conditions as the previous section.

The polar diagrams obtained for $f_{B}$ can be seen in Fig. 7. Adopting again the transfer function (7), with $l=B$, it yields the estimated parameters $\{\kappa, a, \alpha, b, \beta\}$ depicted in Fig. 8. These parameters can be approximated also through the power expressions (10) leading to the results shown in Table 2. Function $f_{B}$ reveals a behavior with similarities to the previous case, with exception of the gain $\kappa$ that has a small variation with $\Delta p$.

The study of the fitness function $f_{C}$ follows an identical strategy. Nevertheless, it results a different type of output and transfer function as can be seen in Figs. 9 and 10. The output is less sensitive and responds only after a delay of several generations. Consequently, this optimization GA leads to a different polar diagrams for $H(\mathrm{j} w)$ (see Fig. 11). In this case the median $H(\mathrm{j} w)$ can be approximated by

$G_{C}(\mathrm{j} w)=\frac{\kappa \mathrm{e}^{-\mathrm{j} w T}}{[(\mathrm{j} w / a)+1]^{\alpha}[(\mathrm{j} w / b)+1]^{\beta}}$

leading to a minimum phase system in contrast to the previous cases.
In this simulation only $\kappa$ (Fig. 12) follows clearly Eq. (10), resulting the estimated values depicted in Table 3. The other parameters reveal a kind of noisy behavior and no clear trendlines emerge. Nevertheless, in order to easy comparisons it was decided to maintain the parameters estimation through (10) leading to exponents $\gamma_{2}$ close to zero.

\section{Conclusions}

This paper analyzed the signal propagation and the dynamic phenomena involved in the time evolution of a population of individuals. The study was established on the basis of a simple GA optimization using three different functions. While the study of GA schemes has been extensively studied, the influence of perturbation signals over the operating conditions is not well known.

From the results can be concluded that the optimization functions and the time duration of perturbation have a strong influence upon the behavior of the GA while the value adopted for the mutation probability seems to be less significant.

Bearing these ideas in mind, a FC calculus perspective was introduced in order to develop approximating transfer functions analytically simple yet meaningful. It was shown that fractional-order models capture phenomena and properties that classical integer-order sometimes overlook due to the approximation requirement for the introduction of a large number of parameters that makes things unclear.

Table 3

Parameters of $\gamma_{i}, i=\{1,2\}$ for $\{\kappa, T, a, \alpha, b, \beta\}$ approximation of $f_{C}$ optimization function

\begin{tabular}{lllrr}
\hline$\Delta p$ & $\kappa$ & & \multicolumn{1}{c}{} \\
\cline { 2 - 3 } & $\gamma_{1}$ & $\gamma_{2}$ & $\gamma_{1}$ & \\
\hline (a) $\kappa, T$ parameters vs. $\Delta p$ & & & & \\
0.03 & 28328 & -0.51 & 0.34 & \\
0.04 & 21467 & -0.51 & 57.22 & 0.02 \\
0.05 & 18418 & -0.53 & 87.80 & -0.36 \\
\hline
\end{tabular}

(b) $a, \alpha, b, \beta$ parameters vs. $\Delta p$

\begin{tabular}{|c|c|c|c|c|c|c|c|c|}
\hline \multirow[t]{2}{*}{$\Delta p$} & \multicolumn{2}{|c|}{$a$} & \multicolumn{2}{|l|}{$\alpha$} & \multicolumn{2}{|l|}{$b$} & \multicolumn{2}{|l|}{$\beta$} \\
\hline & $\gamma_{1}$ & $\gamma_{2}$ & $\gamma_{1}$ & $\gamma_{2}$ & $\gamma_{1}$ & $\gamma_{2}$ & $\gamma_{1}$ & $\gamma_{2}$ \\
\hline 0.03 & 0.58 & -0.38 & 11.71 & -0.32 & 0.45 & -0.04 & 9.65 & 0.23 \\
\hline 0.04 & 0.16 & -0.35 & 1.50 & -0.35 & 0.61 & -0.23 & 19.72 & -0.16 \\
\hline 0.05 & 0.25 & -0.18 & 34.51 & -0.21 & 0.88 & -0.27 & 3.09 & -0.08 \\
\hline
\end{tabular}




\section{Acknowledgment}

This paper is partially supported by the grant Prodep III (2/5.3/2001) from FSE.

\section{References}

[1] T. Bäck, U. Hammel, H.-P. Schwefel, Evolutionary computation: comments on the history and current state, IEEE Trans. Evolutionary Comput. 1 (1) (1997) 3-17.

[2] B. Ross, Fractional Calculus and its Applications, Lecture Notes in Mathematics, vol. 457, Springer, Berlin, 1974.

[3] K.S. Miller, B. Ross, An Introduction to the Fractional Calculus and Fractional Differential Equations, Wiley, New York, 1993.

[4] K.B. Oldham, J. Spanier, The Fractional Calculus: Theory and Application of Differentiation and Integration to Arbitrary Order, Academic Press, New York, 1974.

[5] S.G. Samko, A.A. Kilbas, O.I. Marichev, Fractional Integrals and Derivatives: Theory and Applications, Gordon and Breach Science Publishers, New York, 1993.

[6] A. Gement, On fractional differentials, Proc. Philos. Mag. 25 (1938) 540-549.

[7] A. Oustaloup, La Commande CRONE: Commande Robuste d'Ordre Non Intier, Hermes, 1991.

[8] A. Oustaloup, La Dérivation Non Entier: Théorie, Synthèse et Applications, Editions Hermes, 1995.

[9] C.G. Koh, J.M. Kelly, Application of fractional derivatives to seismic analysis of base-isolated models, Earthquake Eng. Struct. Dyn. 19 (1990) 229-241.

[10] J.A. Tenreiro Machado, Analysis and design of fractionalorder digital control systems, J. System Anal.-ModellingSimulation 27 (1997) 107-122.
[11] J.A. Tenreiro Machado, System modeling and control through fractional-order algorithms, FCAA-J. Fractional Calculus Appl. Anal. 4 (2001) 47-66.

[12] I. Podlubny, Fractional Differential Equations, Academic Press, San Diego, 1999.

[13] B.M. Vinagre, I. Petras, I. Podlubny, Y.Q. Chen, Using fractional order adjustment rules and fractional order reference models in model-reference adaptive control, Nonlinear Dyn. 1-4 (29) (2002) 269-279.

[14] P.J. Torvik, R.L. Bagley, On the appearance of the fractional derivative in the behaviour of real materials, ASME J. Appl. Mech. 51 (1984) 294-298.

[15] O.P. Agrawal, Solution for a fractional diffusion-wave equation in a bounded domain, Nonlinear Dyn. 29 (1-4) (2002) $145-155$

[16] S. Westerlund, Dead Matter Has Memory! Causal Consulting, Kalmar, Sweden, 2002.

[17] T.J. Anastasio, The fractional-order dynamics of brainstem vestibulo-oculomotor neurons, Biol. Cybern. 72 (1994) 69-74.

[18] Y. Chen, K.L. Moore, Discretization schemes for fractionalorder differentiators and integrators, IEEE Trans. Circuits Systems 49 (3) (2002) 363-367.

[19] A.L. Méhauté, Fractal Geometries: Theory and Applications, Penton Press, Golden, Columbia, 1991.

[20] J.A. Tenreiro Machado, A.M.S.F. Galhano, A statistical perspective to the Fourier analysis of mechanical manipulators, J. Systems Anal.-Modelling-Simulation 33 (1998) 373-384.

[21] L. Figueiredo, J.A. Tenreiro Machado, J.R. Ferreira, Dynamical analysis of freeway traffic, IEEE Trans. Intell. Transp. Systems 5 (4) (2004) 259-266.

[22] K. Deb, Multi-Objective Optimization Using Evolutionary Algorithms, Wiley, New York, 2001.

[23] E.J. Solteiro Pires, J.A. Tenreiro Machado, P.B. de Moura Oliveira, Fractional order dynamics in a GA planner, Signal Process. 83 (11) (2003) 2377-2386. 